\title{
TELESCOPE BIBLIOMETRICS 101
}

\author{
UTA GROTHKOPF \\ European Southern Observatory \\ Karl-Schwarzschild-Straße 2 \\ D-85748 Garching, Germany \\ esolib@eso.org \\ AND \\ JILL LAGERSTROM \\ Space Telescope Science Institute \\ 3700 San Martin Drive \\ Baltimore MD 21218, USA \\ lagerstrom@stsci.edu
}

\begin{abstract}
During recent years, bibliometric studies have become increasingly important in evaluating individual scientists, institutes, and entire observatories. In astronomy, often librarians are involved in maintaining publication databases and compiling statistics for their institutions. In this paper, we present a look behind the scenes to understand who is interested in bibliometric statistics, which methodologies astronomy librarians apply, and what kind of features next-generation bibliographies may include.
\end{abstract}

\section{Introduction}

There are many ways to assess research output, for instance by investigating how many research grants have been received, when and where research has been presented at conferences, or how many students graduated under the supervision of specific researchers. Another common tool is bibliometric studies, i.e., using metrics to measure productivity and impact through publications and citations.

Bibliometric studies have quite some history; studies go at least back to the 1960s when the Science Citation Index was first issued. A large number of papers have been published on this topic in general and more specifically in the context of astronomy.

In this paper, we will focus on the following aspects:

- Bibliometric studies - what are they and who is interested? 
- Linking publications and data - how does this happen, and where can interested users get access?

- Telescope bibliographies - who compiles them, and how? What are the current tools and methodologies?

Finally, we will take a look ahead to see what might be coming next.

\section{Typical bibliometric measures}

Many bibliometric studies use one or several of the following measures. However, they all have some advantages as well as some disadvantages.

- Number of publications: measures productivity, but does not report anything about the impact

- Number of citations: gives information about the impact, but can be inflated due to many reasons, for instance incorrect or incomplete citations, as well as biased citing behavior (authors citing well-known authors rather than young, unknown ones)

- Mean or median cites per paper: this measure is better suited for comparisons of scientists or facilities that have been active for different numbers of years, but it seems to reward low productivity

- 'High-impact papers: this metric has been introduced in astronomy by Juan P. Madrid, then at the STScI, a few years ago. Basically, Madrid used the ADS to retrieve information about the 200 most-cited papers in a given publication year, identified those that were based on observational data and calculated the impact of facilities (telescopes, observatories) of each paper. The results per paper were added, and the facilities ranked by contribution to this set of Top 200 papers. The drawback of this method is that so-called hot topics are favored and can outnumber all other facilities for instance in a year of data release. The method is also time-consuming and, to a certain extent, subjective as the contribution percentage is assigned by the bibliometric researcher

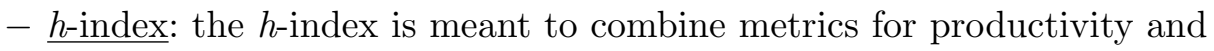
impact (Hirsch 2005); $h$ itself is not suited for comparisons as it does not contain information about the number of years of operation. For comparisons another value, the so-called $m$ parameter, also described by Hirsch, should be used.

All measures have to be applied with greatest care and never in an isolated way as they only shed light on a very limited area of performance of research output. If used for comparisons, several metrics should be applied in parallel to get a more complete picture. 


\section{Users of bibliometric studies}

A wide range of groups are interested in bibliometrics, including instrument scientists, management of observatories, governing bodies and funding agencies who want to

- evaluate the performance of telescopes and instruments

- measure the scientific output from observing programs

- define guidelines for future facilities

- compare in-house facilities with other observatories and telescopes

- interconnect resources, for instance in the context of Virtual Observatory projects

Establishing telescope bibliographies is the prerequisite to bibliometric studies in astronomy; it closes the loop from (a) observing programs carried out by astronomers, (b) data stored in an archive, (c) scientific papers which use the data, and (d) records in telescope bibliography databases that connect the papers with the data through observing programs (program IDs). Archives can be searched by program ID, and all papers published so far that use the data these programs generated can be listed. This procedure assures a maximum return of science benefits from observing proposals, for instance by making archived data easily available to other interested researchers.

Telescope bibliographies can be accessed in various ways. Firstly, a listing has been compiled by the ESO Library and is available on the web ${ }^{1}$; it provides links to the databases of many large observatories. The user interfaces of telescope bibliographies of all major observatories provide the option to search by observation or data set number. If these numbers are noted in the bibliographies' records, a listing of all published articles using the data is just a click away.

For example, in the case of ESO, the public interface allows to search for specific programs, or for papers that use data from certain instruments. The result will be a list of papers for which the facilities that were used are shown, as well as the program IDs. A click on the program ID will take users to the observing schedule from where more information on the proposals can be accessed. The data can be requested, or other papers using the same program can be found.

Another way of finding lists of papers that use observational data from specific observatories is to use the ADS Filters section which can be found in the lower part of the main ADS search screen". The option "All of the following groups" as well as at least one of the listed facilities need to be

\footnotetext{
${ }^{1}$ http://www . eso.org/libraries/publicationlists.html
}

${ }^{2}$ http://adsabs.harvard.edu/abstract_service.html 
Access to literature
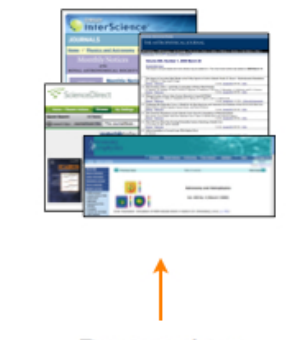

Data archive

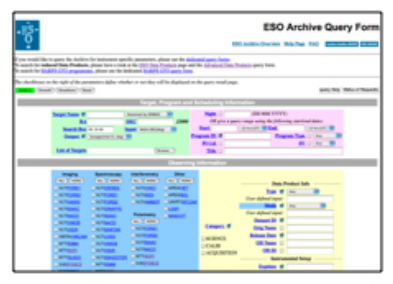

Semi-automated search tool

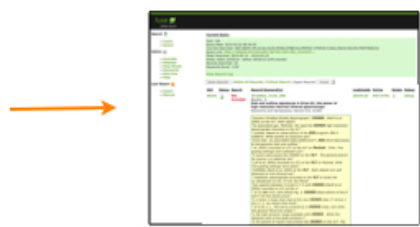

Tags / keywords / programs

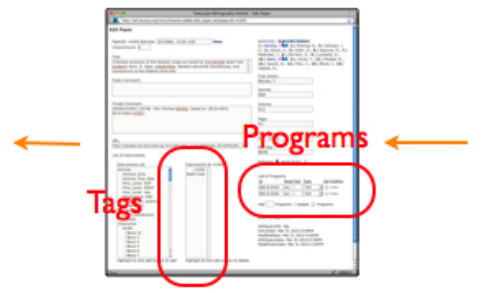

Policy / selection criteria

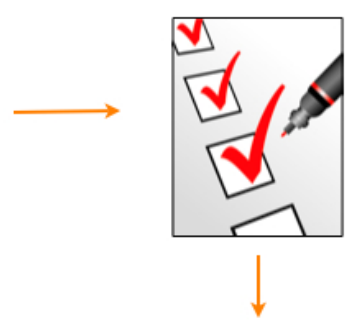

Database

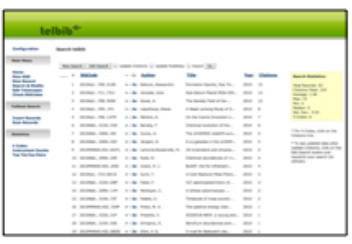

Figure 1. Telescope bibliographies close the loop from published literature to data located in archives, and back.

selected. The records contained in the results set will all have $D$ (for data) links which link back to the underlying observations.

\section{Compiling telescope bibliographies}

In order to compile such literature lists with links to data, or telescope bibliographies, certain prerequisites are necessary (Fig. 1).

Compilers need to have access to the literature. This can be in the form of print versions of scientific journals, or electronic access. The latter can be established by either pulling over PDFs to screen them locally, or by searching full-texts at the publishers' sites. Ideally, a semi-automated search tool is available that helps compilers to pinpoint relevant articles. This will be explained in more detail below. If such a search tool is not in place, PDFs have to be searched one-by-one, or the paper version has to be inspected visually.

Most importantly, a policy needs to be in place that determines which 
papers shall be included in the telescope bibliography, and which are to be excluded. The topic of selection criteria can be handled quite differently among large observatories. Once relevant articles are identified, a database has to be established to host records with bibliographic information about the published papers.

Observatories will have their own individual set of tags and keywords which are assigned to records to describe the content of the papers as well as the facilities that generated the data. Specific information, such as program IDs, will be added at this stage, too. These identifiers link to the observatories' archive, and from there back to the published articles.

\section{Staffing situation, journals screened, and search strategies}

An additional very important "ingredient" needed to establish and maintain telescope bibliographies is staff. In many observatories, librarians are involved in the process, or are even the main person in charge.

In early 2010, Jill Lagerstrom, librarian at STScI, conducted a survey among 16 large observatories (Lagerstrom 2010). A total of 14 institutions replied, namely CFHT, Chandra, FUSE/Galex/IUE, ESO, Gemini, HST/STScI, Isaac Newton Group, Keck, NOAO, NRAO, SDSS, Spitzer, Subaru, and XMM Newton. The survey revealed that large observatories involve between one and four staff members to compile their bibliographies, even though not all of them full-time.

The survey also focused on the journals which are searched by these institutions. Respondents provided a combined list of more than 30 journals which are screened, but surprisingly, only four are searched by all survey participants: $A \xi A, A J, A p J$ and Supplement, as well as MNRAS.

The full-text can be accessed using one or several of the following methods:

- Print journals: screening the full-text by using the print version of journals provides access to the entire text of papers including footnotes, figures, captions, etc. However, it depends on the skills and capabilities of the compilers to detect all instances of relevant keywords and facilities in the text to gather a complete list of published articles

- E-journals (PDFs): this methodology implies retrieving the PDF format of articles from the publishers' web sites and searching them locally. Like with method 1, all sections of papers are screened.

- Journal web sites: many publishers provide an option to search fulltexts of articles directly at their web site. While this functionality can indeed be very useful, it is usually not immediately understandable for users whether this feature actually searches the entire text, or whether 
certain sections are not indexed and can therefore not be searched properly.

- ADS abstracts: at present (May 2010), ADS provides full-text searches only for historic literature; access to recent publications includes only title, abstract, and selected footnotes. If compilers of telescope bibliographies rely on ADS abstract searches for their work, they must be aware that almost inevitably they will miss many relevant papers.

- Author self-reporting: some compilers use reports from authors as an additional or even exclusive means to find papers. It must be noted that, if applied as the sole method for collecting articles, relying on author self-reports will reveal only a fraction of all relevant papers; it is therefore the least recommended way of compiling telescope bibliographies.

The survey also investigated whether compilers of bibliographies use similar search statements, and how elaborate and comprehensive they are in their attempts to identify papers. Not surprisingly, all respondents stated that they search for their observatory's telescopes, typically both the abbreviation as well as the full name. Some are more specific and include instrument names, as well as surveys, archives, or specific science programs (e.g., GOODS). For some institutions, it can be useful to search for geographic locations (Green Bank, Paranal, etc.) or even for concepts (e.g., X-ray).

The final survey question aimed at the biggest challenge compilers face in gathering papers. The majority of respondents stated that their biggest problem is authors who do not provide sufficient details about the source of their data, so that the programs that generated the data cannot be traced without doubt. Others replied that they face problems with restrictive publishers when they access large numbers of articles at their web sites. Many compilers feel that the amount of work time they can dedicate to their respective telescope bibliography is not sufficient in order to complete an exhaustive search. The idea of having a central search option for full-texts at the ADS was ventilated; this would be more time and resources-efficient than duplicating retrieval efforts locally at each observatory, as is currently done.

It became evident that the most difficult part is to define a policy to clearly govern which papers fulfill the criteria for inclusion, and which do not, as well as consistency in applying this policy. Recently, a group has been formed, initiated by the compilers of telescope bibliographies at the Chandra Archive, STScI, and ESO, to provide best practices for maintaining bibliographies, to develop recommendations for using cross-facility 
bibliometrics, and to share solutions to common problems ${ }^{3}$.

\section{Tools of the trade: FUSE and telbib}

While compilers of bibliographies in the past had to rely on their own abilities to retrieve relevant papers by visually scanning the literature, lately some tools have become available to support them in their work. One of these tools is the ESO Full-Text Search tool (FUSE) which has been developed by the ESO Library. Based on an ADS search, FUSE pulls over PDFs from publishers, converts them into text, and searches for keywords and text strings chosen by the individual observatory. If keywords are detected, they are shown in context (two lines of text) for inspection by the compilers. The excerpts often reveal immediately whether the highlighted paper should be investigated in more detail, or whether the keywords are used in a context that is not relevant for the compilation of the telescope bibliography (Fig. 2).

It is important to note that FUSE can only be used as a help application in order to spot potential candidates for inclusion in telescope bibliographies. By no means does it replace the human (intellectual) investigation that is necessary in order to determine whether an observatory's selection criteria for inclusion in the bibliography are actually met by the paper.

Another important tool is telbib, a content management software also developed by the ESO Library. It is used to establish and maintain a database of records with bibliographic information about papers pertaining to a telescope bibliography, to store additional metadata (e.g., tags to describe observing facilities and programs), and to generate reports and statistics. The librarians' user interface provides access to records through a large variety of search criteria (Fig. 3). A public user interface is also available, even though with less detailed query features ${ }^{4}$. Using telbib, bibliography compilers can import bibliographic information and other metadata from the ADS, including author affiliations and number of citations. Observatory-defined tags and keywords can then be added, as well as specific information about observing programs, modes (e.g., visitor or service mode) and types (normal, large, guaranteed time observing types, etc.) .

Telbib is also used to generate reports and statistics either on demand or regularly, for instance the "Basic ESO Statistics" document ${ }^{5}$ that is available from the Libraries' home page ${ }^{6}$. It provides information on ESO publication and citation statistics at large as well as for specific instru-

\footnotetext{
${ }^{3}$ http://groups.google.com/group/astrobib/

${ }^{4}$ http://www.eso.org/libraries/telbib.html

${ }^{5}$ http://www .eso.org/sci/libraries/edocs/ESO/ESOstats.pdf

${ }^{6}$ http://www.eso.org/libraries/
} 


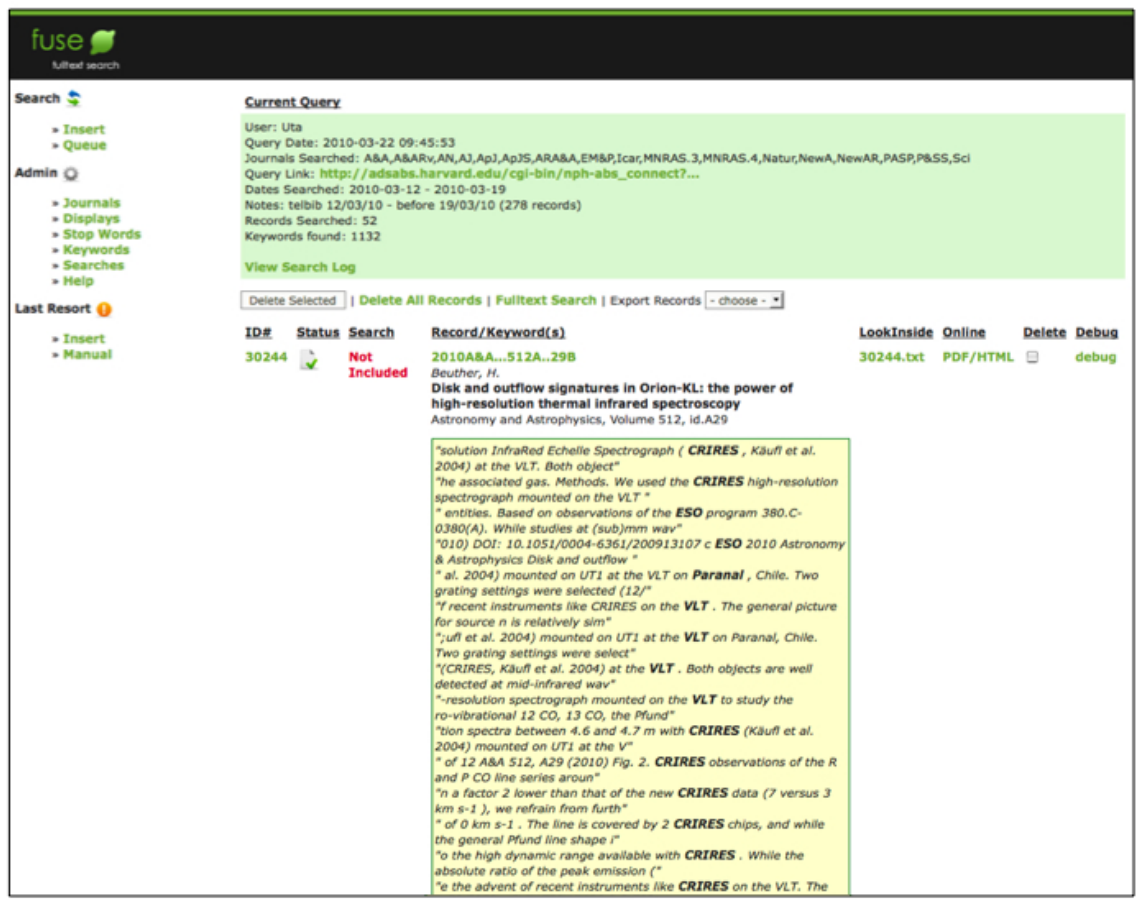

Figure 2. Screenshot of the ESO Full-Text Search tool (FUSE).

ments, identifies the "ESO Top 20" papers, and looks at ESO publications in comparison with other large observatories.

A more detailed description of FUSE and telbib including their development and features can be found in Erdmann \& Grothkopf (2010).

FUSE is currently in use at ESO, STScI, Gemini, Subaru, and the Carnegie Observatories. The libraries of SAAO (South African Astronomical Observatory) in Cape Town, South Africa, and IUCAA (Inter-University Centre for Astronomy and Astrophysics) in Pune, India, are in the process of installing the software. Because of this obvious duplication of efforts by conducting searches locally, recently the idea of creating a central search facility at the ADS has been discussed. Compilers of bibliographies could then run tailored searches at the ADS without the need to download PDFs locally for inspection. The search feature would only highlight potentially relevant matches by showing brief excerpts of text; access to the full-texts would still be governed by the publishers through subscriptions. 


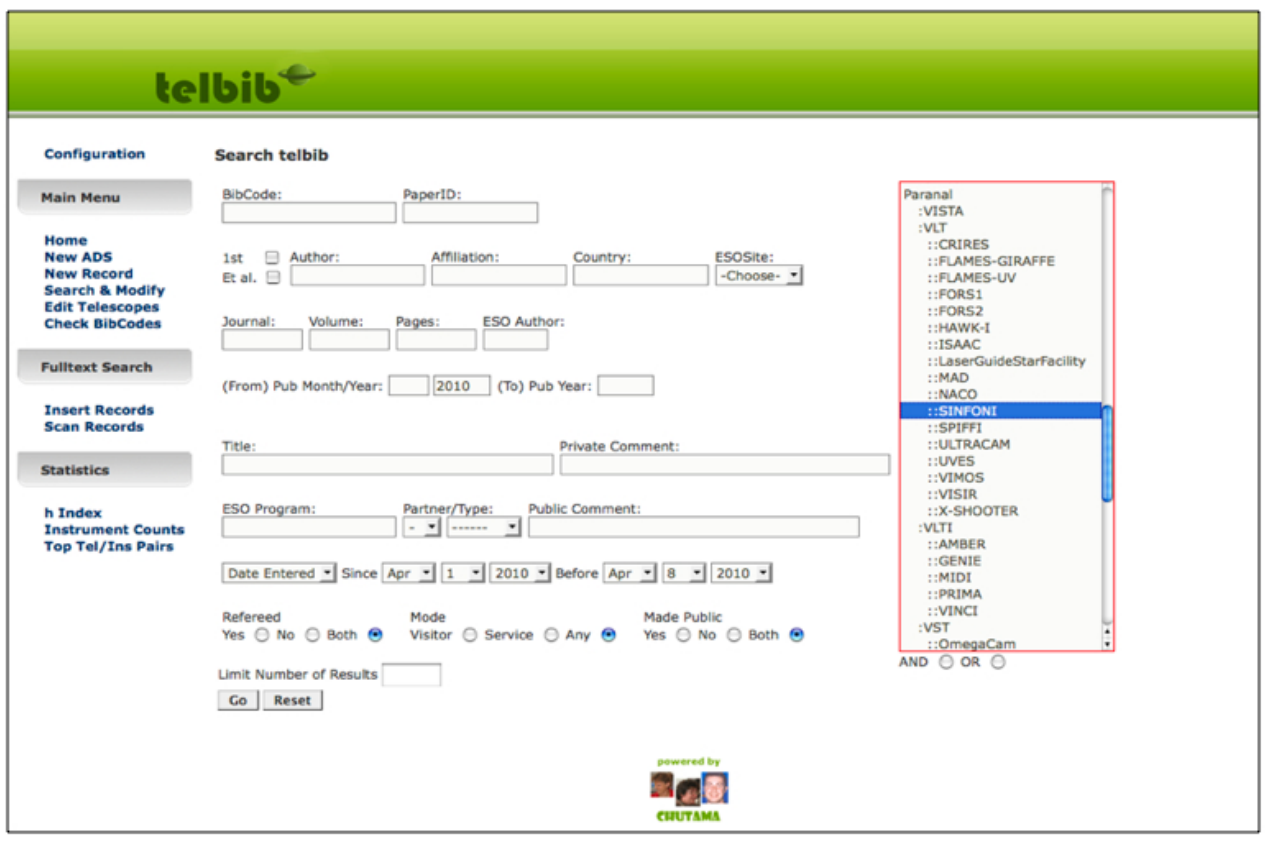

Figure 3. Screenshot of the ESO telbib tool.

\section{Beyond citations}

As already mentioned, studying citations to measure scholarly output is problematic, despite their wide-spread use for this purpose. Lists of citations are often incomplete, references are incorrect, various abbreviations for the same journal are used in parallel and are sometimes not attributed to the correct publication, and the typical citing behavior often favors wellknown researchers instead of referencing younger, unknown authors.

Are there other, possibly better ways of evaluating scientific impact? Compilers of telescope bibliographies are experimenting with other measures. Some of them are described below.

It is well known that the ADS provides information about citations for each paper in their database; these are limited to those citing papers that also reside in the ADS database, hence they are not entirely complete. However, in the area of astronomy this is a minor disadvantage as the ADS coverage of papers is extremely large.

Few users are aware that ADS also gathers information about so-called 
Reads. Reads are defined as those instances where users access more detailed information than is available in the standard brief results list displayed after an ADS search. Hence, a Read can mean that a user looked at the abstract, the list of references, or the citing papers. Also any time the electronic version of an article is accessed, be it in PDF or in HTML format, counts as a Read.

The number of Reads typically differs considerably from the number of citations an article has gathered. More importantly, the distribution of Reads versus citations as a function of years reveals that many papers which are not cited frequently anymore still are used regularly by the community.

An even more telling statistic about actual usage could be obtained by limiting Reads to the actual request of full-texts, i.e., PDFs and HTML downloads. Such numbers are traced by the ADS, but in contrast to Reads, they cannot be retrieved through the ADS user interface. However, the ADS team is always helpful in providing such statistics on request.

Another project that operates along the same line of thought is Citebase $^{7}$. Citebase is a citation index that "harvests pre- and post-prints (mostly author self-archived)" from arXiv and other repositories and lists them together with papers that cite them. In addition, they provide information about downloads by country, by date and by organization. Unfortunately, they are still in experimental stage, and users are cautioned not to use the results for academic evaluation.

A newcomer in the area of evaluating research impact is the idea to look at social networking platforms and forum discussions. In astronomy CosmoCoffee $^{8}$ might be one such discussion group that reveals the community's interest in specific papers. Registered users refer to manuscripts posted on arXiv and suggest discussion among their peers. In order to track impact, bibliography compilers could track which arXiv preprints are discussed so that these papers can be linked to the final versions once they are published, and the number of comments and discussion threads specific papers generate can be traced.

\section{Ongoing projects}

In order to provide even more informative reports and to anticipate future interests of management and funding organizations, it is desirable to import further metadata from the ADS, for instance subject terms and keywords (to gain knowledge about the specific subject areas in which users of observational data publish), full information of citing papers (in order to eliminate self-citations), all other available links (e.g., Digital Object Iden-

\footnotetext{
${ }^{7}$ http://www. citebase.org/

${ }^{8}$ http://www. cosmocoffee.info/
} 
tifiers (DOI), eprint IDs to establish links between eprints and published papers), as well as the author gender. The latter might be of interest to researchers involved in topics such as "Women in Astronomy"; identifying an author's gender may be feasible in future with the help of initiatives like ORCID (Open Researcher and Contributor ID) ${ }^{9}$ that are hoped to solve the name ambiguity problem in scholarly research.

It is also intended to establish additional links, for instance between telescope bibliography records and press releases that feature specific papers, as well as record detailed information about the observing dates so that the delay from data acquisition to the publication can be computed.

\section{Conclusion}

The task of compiling telescope bibliographies has evolved considerably during recent years, providing ever more detailed and sophisticated information about the papers contained in the databases of large observatories. This trend will continue in the future as management and funding agencies rely increasingly on bibliometric studies in order to evaluate research output. It is important that compilers collaborate and exchange ideas and solutions to common problems in order to develop best practices and recommendations for establishing, maintaining, and using telescope bibliographies in a standardized, reproducible manner.

\section{Acknowledgments}

The ESO Telescope Bibliography (telbib) and Full-Text Search tool (FUSE) use NASA's Astrophysics Data System (ADS) Abstract Services. The authors wish to thank the ADS team for their excellent service to the entire community.

\section{References}

1. Erdmann, C. \& Grothkopf, U., 2010: Next generation bibliometrics and the evolution of the ESO Telescope Bibliography, in: Library and Information Services in Astronomy VI, Isaksson, E., Lagerstrom, J., Holl, A. and Bawdekar, N. (eds.), Astronomical Society of the Pacific, San Francisco, ASP Conference Series 433, p. 81

2. Hirsch, J.E., 2005: An index to quantify an individual's scientific research output, Proc. Nat. Acad. Sci., 102, 16569, DOI 10.1073/pnas.0507655102

3. Lagerstrom, J, 2010: Comparison of methods for creating telescope bibliographies, in: Library and Information Services in Astronomy VI, Isaksson, E., Lagerstrom, J., Holl, A. and Bawdekar, N. (eds.), Astronomical Society of the Pacific, San Francisco, ASP Conference Series 433, p. 89

${ }^{9}$ http://www . orcid.org/ 